\title{
Description and molecular characterization of a new species of tarantula, Pamphobeteus verdolaga, from Colombia (Araneae: Mygalomorphae: Theraphosidae)
}

\author{
Yeimy Cifuentes', Sebastián Estrada-Gomez², Leidy Johana Vargas-Muñoz \& Carlos Perafán ${ }^{*}$
}

\author{
'Laboratório Especial de Ecologia e Evolução, Instituto Butantan. Avenida Vital Brazil 1500, 05503-900 São Paulo \\ SP, Brazil. E-mail: yelicigi@gmail.com \\ 2 Programa de Ofidismo/Escorpionismo - Serpentario, Facultad de Ciencias Farmacéuticas y Alimentarias, Universidad \\ de Antioquia. Medellín, Colombia. E-mail: sebastian.estrada@udea.edu.co \\ ${ }^{3}$ Facultad de Medicina, Universidad Cooperativa de Colombia, Sede Medellín. E-mail: leidy.vargasmu@campusucc.edu.co \\ ${ }^{4}$ Sección Entomología, Facultad de Ciencias, Universidad de La República. Iguá 4225, Montevideo, Uruguay. \\ E-mail: caperafanl@gmail.com \\ ${ }^{*}$ Corresponding Author.
}

\begin{abstract}
A new species of Pamphobeteus Pocock, 1901 is described from the Colombian Andes, Medellín, Antioquia. The biochemistry and molecular characteristics of the venom of this new species (previously identified as Pamphobeteus aff. nigricolor) has been already described. A detailed taxonomic study, comparing this species with holotypes and additional material of Pamphobeteus species, allowed us to recognize it as new, and to describe it here as Pamphobeteus verdolaga sp. nov. The male of $P$. verdolaga sp. nov. is distinguished by the palpal bulb with broad embolus, poorly developed apical keel $(\mathrm{A})$, prolateral inferior keel $(\mathrm{PI})$ and prolateral accessory keel (PAC) present but poorly developed, and retrolateral keel (R) of similar length as A. Females are distinguished by the morphology of spermatheca with a wide base and very short oval seminal receptacles, which are curved toward the center. This is the thirteenth species described in Pamphobeteus and the sixth species reported from Colombia. The species description is complemented by a molecular characterization of a partial CO1 sequence.
\end{abstract}

KEY WORDS. CO1 sequence, Medellín, Neotropical, Theraphosinae.

Pamphobeteus Pocock, 1901 comprises 12 species of tarantulas, distributed in northern South America (WORLD SPIDER Catalog 2016). They are mainly distributed on the Andean region, with high diversity in Colombia, although they are also found in the Amazon rainforest (BerTani et al. 2008). Pamphobeteus includes large terrestrial species, usually dark colored with violet shades. They inhabit burrows or natural cavities.

Males of Pamphobeteus can be distinguished morphologically by the presence of a palpal bulb with a concave-convex embolus, spoon-shaped, with a well developed apical keel (A) that extends up to the edge of the embolus, a well developed retrolateral keel (R), and a prolateral superior keel (PS) (BERTANI et al. 2008). Females can be distinguished by the spermatheca with two seminal receptacles largely fused at the base and apically elongated (Pérez-Miles et al. 1996). Pamphobeteus males and females resemble Xenesthis Simon, 1891 but they can be distinguished from those by having the scopulae on metatarsi IV restricted to the apical portion (fully extended on Xenesthis) (BERTANI et al. 2008).

The biochemistry and molecular characteristics of the venom Pamphobeteus were described by Estrada-Gomez et al. (2013) based on specimens from Medellín, Colombia, which had been identified as Pamphobeteus aff. nigricolor. A detailed taxonomic study, and the comparison of this specimens with holotypes, additional specimens, and the original descriptions of Pamphobeteus species, allowed us to recognize that the males from Medellín do not belong to Pamphobeteus nigricolor (Ausserer, 1875) and differ from all other species of the genus in the morphology of the palpal bulb. Based on this comparison we have determined these specimens as a new species, which is diagnosed and described as Pamphobeteus verdolaga sp. nov. The species description is complemented by a molecular characterization of a partial CO1 sequence, the first to be sequenced for the genus. 


\section{MATERIAL AND METHODS}

All measurements are given in millimeters ( $\mathrm{mm})$. Body and legs measurements were taken with a vernier caliper; legs and palp measurements were taken in dorsal view along the central axis of the left-side limbs. Others measurements were taken using an ocular micrometer. The terminology used to described the palpal organ keel follows BERTANI (2000) and for the urticating setae follows Cooke et al. (1972). The number and disposition of spines were enumerated from the anterior third to the posterior third, modified from Petrunkevitch (1925). The geographic coordinates and altitude data (meters above sea level: $\mathrm{m}$ asl) were referenced by GPS, Datum WGS84, or determined using the Instituto Geográfico Agustín Codazzi Gazetter (http:// www.igac.gov.co/digeo/app/index.html). The coordinates are given in UTM notation. The distribution map was produced using SimpleMappr (SHorTHouse 2010).

Holotype material is deposited at the ICN Arachnological Collection, Bogotá, Colombia and paratypes are in the Entomological Collection and Serpentarium of the Universidad de Antioquia, Medellín, Colombia, stored in 70\% ethanol.

Abbreviations: (A) apical keel; (ALE) anterior lateral eyes; (AME) anterior median eyes; (D) dorsal; (OQ) ocular quadrangle, including lateral eyes; $(\mathrm{P})$ prolateral; (PAc) prolateral accessory keel; (PI) prolateral inferior keel; (PME) posterior median eyes; (PMS) posterior median spinnerets; (PLE) posterior lateral eyes; (PLS) posterior lateral spinnerets; (PS) prolateral superior keel; (R) retrolateral; $(\mathrm{V})$ ventral. Institutions: $(\mathrm{BMNH})$ British Museum of Natural History, London, England; (CEUA) Entomological Collection, Universidad de Antioquia, Medellín, Colombia;(IBSP) Instituto Butantan, São Paulo, Brazil; (ICN) Instituto de Ciencias Naturales, Universidad Nacional de Colombia, Bogotá; (MNHN) Muséum National d'Histoire Naturelle, Paris, France. (MNRJ) Museu Nacional do Rio de Janeiro. (QCAZ) Quito-Católica-Zoología, Museum of Zoology, Pontificia Universidad Católica del Ecuador; (SMF) Senckenberg Museum of Frankfurt, Germany; (SUA) Serpentarium, Universidad de Antioquia, Medellín, Colombia; (UDENAR) Universidad de Nariño, Pasto, Colombia.

Material examined of other species. Pamphobeteus antinous: male holotype from Madre de Dios, Bolivia (BMNH 1895-11-92). Pamphobeteus crassifemur: 2 males from Porto Velho, Rondônia, BRAZIL (IBSP 10324), (IBSP 11132). Pamphobeteus grandis: male holotype from Embira river, Acre, Brazil (MNRJ 34). Pamphobeteus ferox: female holotype from Nueva Granada, Colombia (BMNH 1890-7-1-376); 1 male from Cundinamarca, Colombia, road to Tena Km. 47, 1100 m asl, 12 Aug 2002 (ICN-Ar 8034); 1 female from Mesitas, Cundinamarca, Colombia (ICN-Ar 373). Pamphobeteus fortis: female holotype from Bogotá, Colombia (BMNH 1890-7-1-368). Pamphobeteus insignis: male holotype from Cauca, Colombia (BMNH 1895-6-23-2); 1 male from Calarcá, Quindío, Colombia, C. Perafán leg. (ICN-Ar 8037); 2 males from Pasto, Nariño, Colombia (UDENAR). Pamphobeteus nigricolor: male and female types from Nueva Granada, Colombia (BMNH); 1 male and 1 female from Piedracampana, Santamaría, Boyacá,
Colombia, 490 m asl, 14 May 2009, E. Florez \& D. Luna leg. (ICNAr 4165). Pamphobeteus ornatus: male holotype from Valle del Cauca, Colombia, Dagua river (BMNH 1896-3-15-5). Pamphobeteus petersi: male holotype (SMF 40567) and female paratype (SMF 40569) from Ecuador and Peru; Pamphobeteus ultramarinus: male holotype (SMF 38594) and female paratype (SMF 38555) from Ecuador; Pamphobeteus vespertinus: male holotype from Los Puentes, Ecuador (MNHN).

Molecular protocol. Genomic DNA were extracted from one female (SUA 149) using the DNA BLOOD and TISSUE kit from QIAGEN (Qiagen,Valencia, CA). The concentration of the extracted DNA was $10.4 \mathrm{ng} / \mathrm{uL}$ with a relation $260 / 280$ of 1.97 . DNA amplification was performed using the polymerase chain reaction (PCR) for the mtDNA "barcoding" gene regions of the CO1. To amplify the CO1 gene region, we used the oligos C1J1751_Forward GAGCTCCTGATATAGCTTTTCC and C1-N-2776 REVERSE GGATAATCAGAATATCGTCGAGG previously reported amplifying CO1 regions from other Theraphosidae (LONGHORN et al. 2007, Hamilton et al. 2011). The amplification was performed using a mixture of $10 \mathrm{ng}$ of purified DNA and oligos at a concentration of $0.2 \mu \mathrm{M}$. The amplification program used was: $95^{\circ} \mathrm{C} 120$ seconds, $95^{\circ} \mathrm{C} 30$ seconds, $52^{\circ} \mathrm{C} 30$ seconds, $72^{\circ} \mathrm{C} 110$ seconds ( 35 cycles), $72^{\circ} \mathrm{C} 10$ minutes. The amplified product was purified from the gel and submitted to capillary sequence.

\section{TAXONOMY}

\section{Theraphosidae Thorell, 1869 \\ Pamphobeteus Pocock, 1901 Pamphobeteus verdolaga Cifuentes, Perafán \& Estrada-Gomez sp. nov.}

Figs. 1-11

Pamphobeteus aff. nigricolor: Estrada-Gomez et al., 2013: 301. urn:Isid:zoobank.org:act:7D7D3E7C-A485-4908-99C7-700944B790E9

Diagnosis. Male differs from known Pamphobeteus species by having palpal bulb with broad embolus, poorly developed apical keel (A) and prolateral inferior keel (PI), retrolateral keel (R) of similar length to A, and a prolateral accessory keel (PAc). Prolateral superior keel (PS) and R well developed (Figs. 1-7). Females have spermathecae with a wide base and very short seminal oval receptacles, curved toward the center (Fig. 9). The palpal bulb of Pamphobeteus verdolaga sp. nov. resembles that of P. antinous Pocock, 1903; P. crassifemur Bertani, Fukushima \& Silva, 2008; P. grandis Bertani, Fukushima \& Silva, 2008; P. ultramarinus Schmidt, 1995; and P. vespertinus (Simon, 1889) by the broad embolus. But, all of them present palpal bulb with $\mathrm{R}$ shorter than A (R similar to A on P. verdolaga sp. nov.; Figs. 2, 6).

Description. Male holotype (ICN-Ar 8061) (Fig. 10): total length, not including chelicerae or spinnerets 46.87 , carapace length 22.32, width 20.48. Anterior eye row procurved, posterior recurved. Eyes and interdistances: AME 0.45, ALE 0.60, PME 0.45, PLE 0.70, AME-AME 0.50, AME-ALE 0.30, ALE-ALE 1.85, PME-PME 


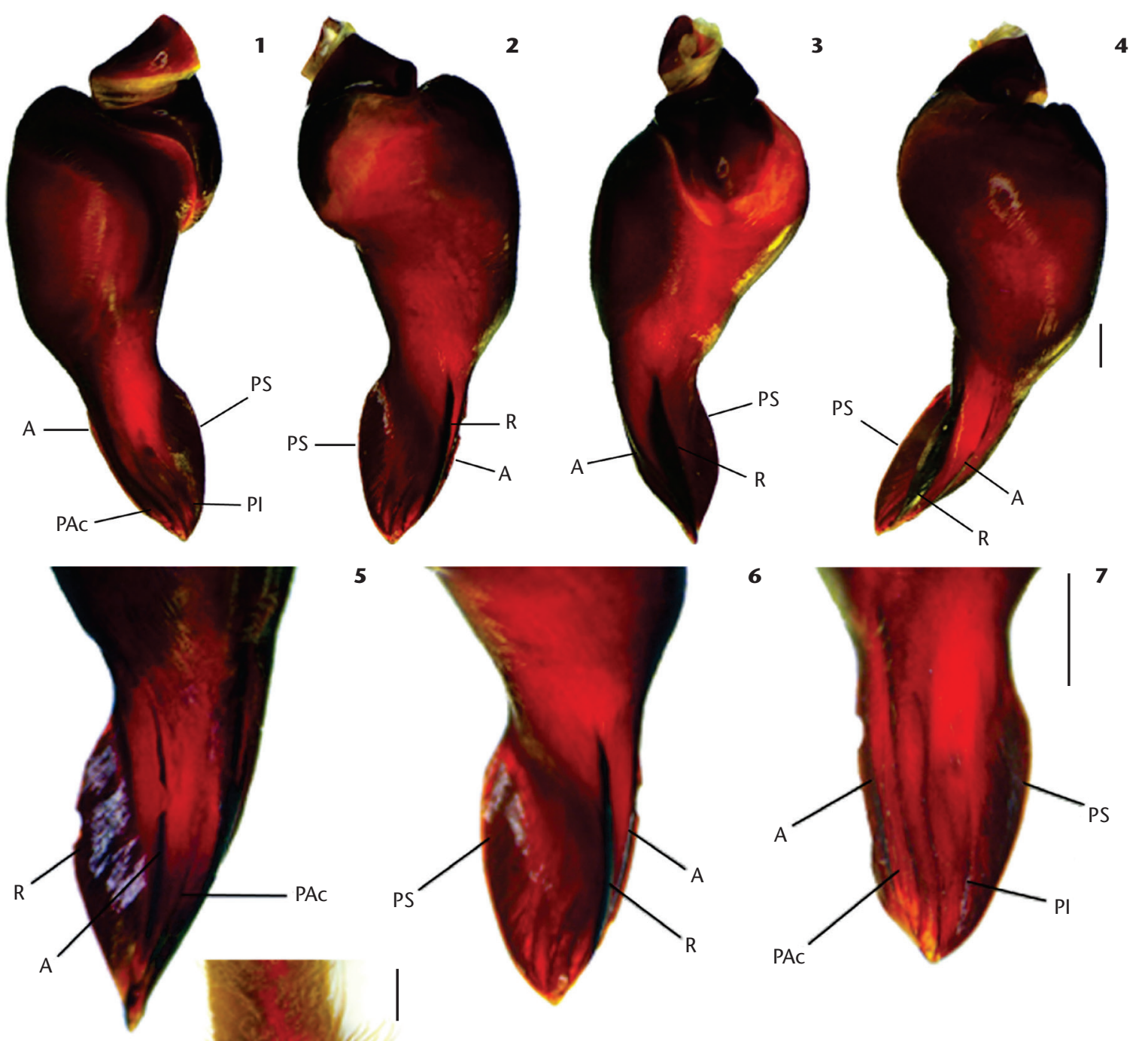

8

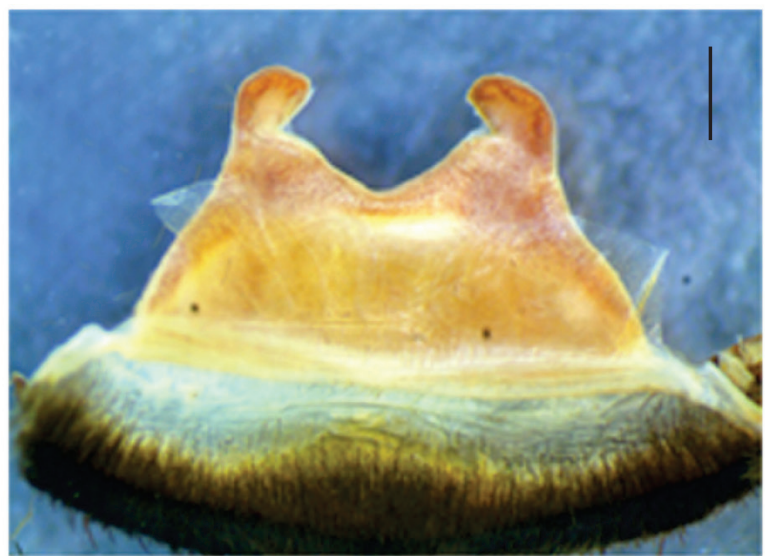

9

Figures 1-9. Pamphobeteus verdolaga sp. nov.: (1) palpal bulb prolateral view, (2) retrolateral view, (3) dorsal view, (4) ventral view; (5-7) detail of distal palpal bulb: (5) prolateral-dorsal view, (6) retrolateral view, (7) prolatero-ventral view; (8) male tibial apophysis, left leg l; (9) spermatheca, ventral view. Scale bar: $1 \mathrm{~mm}$. 
1.35, PME-PLE 0.1, PLE-PLE 2.05, AME-PME 0.2, ALE-PLE 0.6. OQ slightly elevated sub-rectangular, length 2.15 , width 2.90. Clypeus 0.45 . Fovea transverse oval, width 2.25 . Cephalic region slightly elevated, thoracic region wide and striated. Chelicerae with left/ right 11/13 well-developed teeth on furrow promargin, and left/ right $47 / 53$ small teeth on the proximal area of furrow. Intercheliceral tumescense absent. Labium length 2.30, width 3.45, with 83 cuspules. Maxillae left/right with 132/141 cuspules, located at the anterior inner corner. Labio-sternal junction broad. Sternum length 9.40, width 7.60, with three pairs of oval sigillas; each of them separated approximately the length of its diameter from the edge of the sternum. Superior tarsal claws with small teeth on axis central: I, three very small teeth; II, without claws; III, 7 very small teeth; IV, five teeth. Tarsal scopulae: I-IV densely scopulated and entire. Metatarsal scopulae extent: I-III densely scopulated, I-II over distal half, III on distal 1/3; IV slightly scopulated, on distal $1 / 4$ apical.

Tibial apophysis (Fig. 8): ventral apophysis with two well developed branches, retrolateral branch twice larger than prolateral branch. Flexion of metatarsus I between branches.

Copulatory organ (Figs. 1-7): palpal bulb with broad embolus concave-convex aspect, spoon-shaped. Embolus with apical keel (A) and prolateral inferior keel (PI) poorly developed, prolateral superior keel (PS) and retrolateral keel (R) well developed; R length similar to A. Prolateral accessory keel (PAc) present (Figs. 1-7).

Urticating setae: type I and III present. PMS well-developed, length 2.3, PLS normal, basal segment length 4.0, medial 2.90, distal 4.60.

Spination (proximal to distal). Femur, palp: OV, OD, 0-02P, OR; I: OV, OD, OP, OR; II: OV, OD, 0-0-2P, OR; III: OV,0-0-1D, OP, 0-0-1R; IV: OV, OD, OP, OR. Patella, palp: OV, OD, OP, OR; I: OV, OD, OP, OR; II: OV, OD, 0-1-OP, OR; III: OV, OD, 0-2-0P, 0-1-OR; IV: OV, OD, 0-1-OP, 0-1-OR. Tibia, palp: OV, OD, 2-3-2P, OR; I: 0-1-OV, OD, 0-2-OP, OR; II: 0-1-3V, OD, 1-1-2P, OR; III: 1-2-2V, OD, 1-2-2P, 1-1-1R; IV: 0-1-2V, 0D, 2-1-2P, 1-2-1R. Metatarsus, I: 0-0-1V, OD, OP, 0R; II: 1-3-3V, 0D, 0-1-1P, 1-0-0R; III: 0-1-3V, 0-0-2D, 4-4-2P, 1-0-1R; IV: 4-7-3V, 0-0-2D, 2-2-1P, 1-0-2R. Tarsus, palp and legs 0.

Legs and palpal segments lengths: leg I: femur 19.9/ patella 10.3/tibia 14.9/metatarsus 15.4 /tarsus 9.5/total 70.0; II: 19.5/9.7/14.3/15.3/9.4/68.2; III: 17.3/8.8/13.2/12.2/9.0/60.5; IV: 20.5/9.4/16.8/24.7/9.2/80.6; palp: 12.8/6.7/11.0/-/3.7/34.2.

Color (in alcohol): cephalotorax and legs brown, femora darker, abdomen dark brown.

Female paratype (ICN-Ar 8062) (Fig. 11): total length, not including chelicerae or spinnerets 50.6, carapace length 21.6, width 23.4. Anterior eye row strongly procurved, posterior strongly recurved. Eyes and interdistances: AME 0.40, ALE 0.55, PME 0.40, PLE 0.75, AME-AME 0.60, AME-ALE 0.60, ALE-ALE 2.10, PME-PME 1.75, PME-PLE 0.3, PLE-PLE 2.45, AME-PME 0.45 , ALE-PLE 0.55. OQ slightly elevated sub-rectangular, length 2.40, width 3.35. Clypeus 0.40. Fovea transverse oval, width 6.13. Cephalic region slightly elevated, thoracic region wide and striated. Chelicerae with left/right 11/12 well-developed teeth on furrow promargin, and left/right 48/46 small teeth on the proximal area of furrow. Intercheliceral tumescense absent. Labium length 2.85 , width 4.45 , with 92 cuspules. Maxillae left/right with 159/152 cuspules, located at the anterior inner corner. Labio-sternal junction broad. Sternum length 9.90, width 7.90, with 3 pairs of oval sigillas; each of them separated approximately the length of its diameter from the edge of the sternum. Superior tarsal claws with small teeth on axis central; I-III with 4 teeth, palp and IV with 6 teeth. Tarsal scopulae: I-IV and palp densely scopulated and entire. Metatarsal scopulate extent: I-III densely scopulated, I-II over distal half, III on distal half, IV slightly scopulated, on distal $1 / 4$ apical. Urticating setae: type I and III present. PMS well-developed, length 2.30, PLS normal, basal segment length 3.90, medial 2.20, distal 4.30.

Spermathecae: two seminal oval receptacles largely fused on a wide sclerotized base. Base twice wider than long. Seminal receptacles very short and curved toward the center.

Spination (proximal to distal). Femur, palp: OV, OD, 0-02P, OR; I: OV, OD, OP, OR; II: 0V, 0D, 0-0-2 P, OR; III: 0V,0-0-1D, OP, 0-0-1R; IV: OV, OD, OP, OR. Patella, palp: 0V, OD, OP, OR; I: OV, OD, OP, OR; II: OV, OD, OP, OR; III: OV, OD, 0-1-OP, OR; IV: OV, OD, OP, OR.Tibia, palp: 2-2-0V, OD, 2-3-2P, 0-1-1R; I: 0-0-3V, OD, 0-2OP, OR; II: 0-1-3V, 0D, 0-2-1 P, OR; III: 1-1-2V, OD, 0-2-1P, 0-2-1R; IV: 0-2-2V, 0D, 0-2-3P, 0-4-1R. Metatarsus, I: 1-0-2V, OD, OP, 0R; II: 1-0-3V, 0D, 0P, 0-0-1R; III: 1-2-2V, 0-0-1D, 1-0-1P, 0-1-1R; IV: 2-4-3V, 0-0-2D, 2-3-2P, 0-1-1R. Tarsus, palp and legs 0.

Legs and palpal segments lengths: leg I: femur 20.4/ patella 11.0/tibia 15.0/metatarsus 13.3 /tarsus 9.7/total 69.4; II: 18.6/10.0/13.5/13.2/8.9/64.2; III: 17.2/9.0/12.4/15.9/8.6/63.1; IV: 19.9/9.7/15.3/21.7/10.1/76.7; palp: 14.2/7.7/11.0/-/9.6/42.5.

Color (alive): cephalotorax and legs brown, violet overtones on the anterior region of cephalotorax, femora greenish dark brown, abdomen dark brown.

Molecular characterization: mitochondrial CO1 partial sequence ( $414 \mathrm{bp}$ ) (this sequence aligned with other theraphosid spiders using GenBank's Standard Protein Blast confirmed the selection): caatactaaccatagcataaatcattcccaaagacccgaaaggttcccgttttccaacagaagaactgataatatgtgaaaccattccaaaacgaggaagaattaaaatatacacttcagggtgtccaaaaaatcaaaataaatgctgaaacaaaaccggatcaccacctccagcaggatcaaaaaaagaagtattaaaatttcgatcagacaacaacatagtaattgctcccgccaacactggaagagataacaacaacaatacagtcgtaattagaacagatcaaacaaacaacggtactcgttctattgttattcctgatctccgcatattcaaaacagttctaataaaatttaccgaacctataatagaagaagcaccagctaaatgcaaagaaaaaattgcaaaatcca

Type material. Male holotype: Colombia, Antioquia: La Estrella [Pueblo Viejo, aprox. $1700 \mathrm{~m}$ asl, Coord. 6.134149 , $\left.-75.646996^{\circ}\right], 12$ Oct 1998 (ICN-Ar 8061). Female paratype: same locality: [6.133458,$\left.-75.649405^{\circ}\right], 18$ Oct 2013, Y. Cifuentes, C. Perafán, S. Estrada and L.J. Vargas leg. (ICN-Ar 8062). Paratypes: Colombia, Antioquia: Jardín [Km. 4 road to Jardín-Río Sucio, aprox. $1700 \mathrm{~m}$ asl, $5.585167^{\circ},-75.796037^{\circ}$ ], 1 female, 17 Sept 2015, Y. Cifuentes, S. Estrada and L.J. Vargas leg. (SUA147); same data (SUA 148) (SUA 149); Caldas [El Canelón, aprox. $1700 \mathrm{~m}$ 

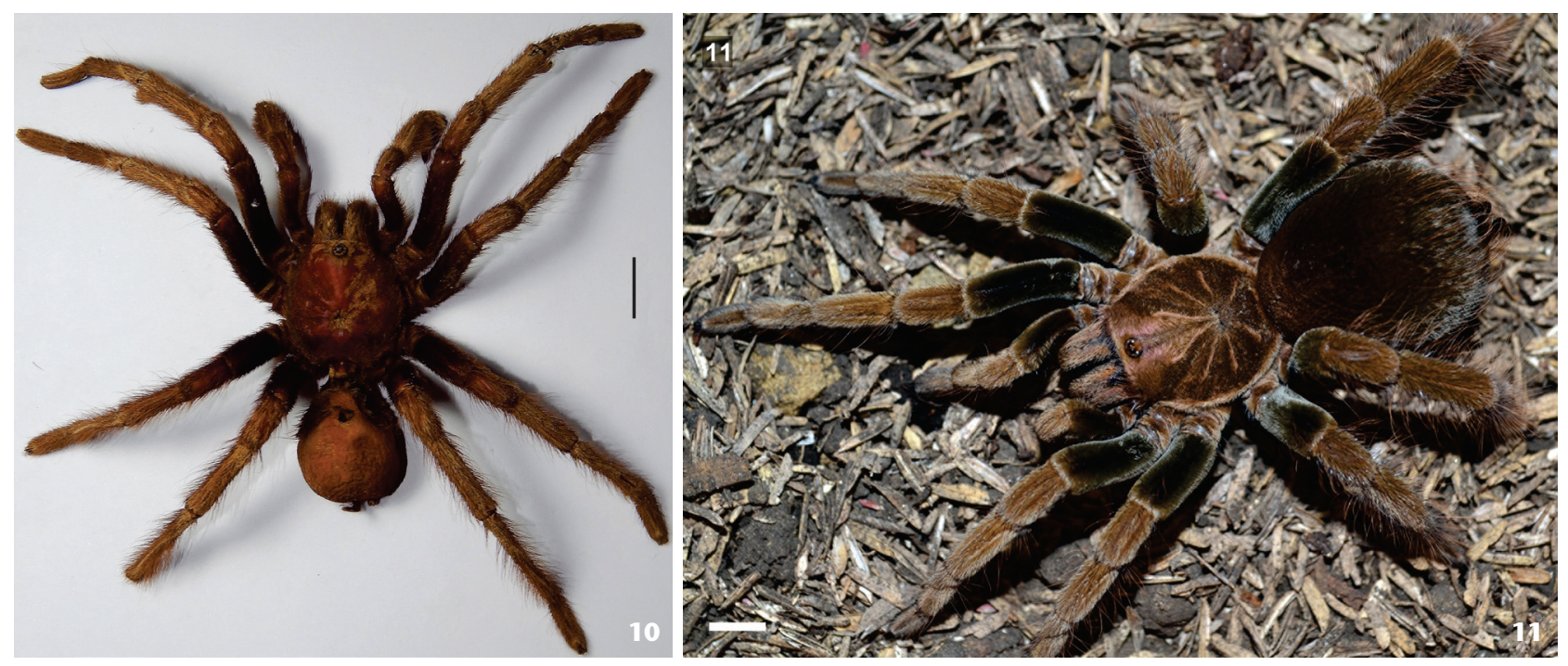

Figures 10-11. Habitus of Pamphobeteus verdolaga sp. nov.: (10) male holotype, scale bar: $10 \mathrm{~mm}$; (11) female paratype.

asl], 1 female, 27 Sep 2004 (SUA 014); Caldas [La Valeria, aprox $1700 \mathrm{~m}$ asl], 1 male, 1 Aug 2006 (SUA 011); Heliconia [aprox. 1800], 1 male, 20 Aug 2014, Ambiental Authority leg. (SUA 128); Caldas [Aguacatala, aprox. $1700 \mathrm{~m}$ asl], 1 female, $10 \mathrm{Jul}$ 2004, M. alvarez leg. (CEUA-Ar 349); Fredonia [aprox. 1800, $5.926667^{\circ},-75.673889^{\circ}$ ], 1 female, 09 Feb 1999 (CEUA-AR 267); Abejorral, 1 male, 02 May 2000, J. Restrepo leg. (CEUA-Ar 228); Amagá [aprox. $1400 \mathrm{~m}$ asl], 1 female, 27 Ago 2000, M. Salgar leg. (CEUA-Ar 269); Amagá [Caserío Los Sánchez], 1 male, 06 Sep 2009, G. Hoyos leg. (CEUA-Ar).

Additional material examined. Colombia, Antioquia: Medellín [aprox. $1450 \mathrm{~m}$ asl], 1 female, 28 Jun 2011, H. León Pérez leg. (SUA 097); Concepción [aprox. $1900 \mathrm{~m}$ asl], 1 female, 10 Jul 2009, Miguel Zapata leg. (SUA 066); Valle de Aburrá, 1 male (SUA 082); Santa Bárbara [aprox. 1800], 1 male (SUA 057) (SUA 059), 1 female (SUA 061).

Distribution. Pamphobeteus verdolaga sp. nov. is known from the Central Cordillera of the Colombian Andes, south of Antioquia Department, around Medellín, approximately between 1400 and $2000 \mathrm{~m}$ asl (Fig. 12).

Etymology. The specific epithet "verdolaga" is a noun in apposition and refers to the main nickname of Football Club Atlético Nacional, one of the football teams based in Medellín. The greenish dark color of the femora of this new species resembles the distinctive green color of the team, which derived from the flags of Antioquia and Medellín, where this species is distributed.

Remarks and affinities. The males of Pamphobeteus are characterized by having palpal bulb with embolus spoon-shaped, whose width varies among species. Some species present the embolus elongated and with narrower concavity: P. augusti (Simon, 1889); P. ferox (Ausserer, 1875); P. fortis (Ausserer, 1875); P. insignis Pocock, 1903; P. nigricolor (Ausserer, 1875); and P. ornatus Pocock, 1903. And the other ones have the palpal bulb with

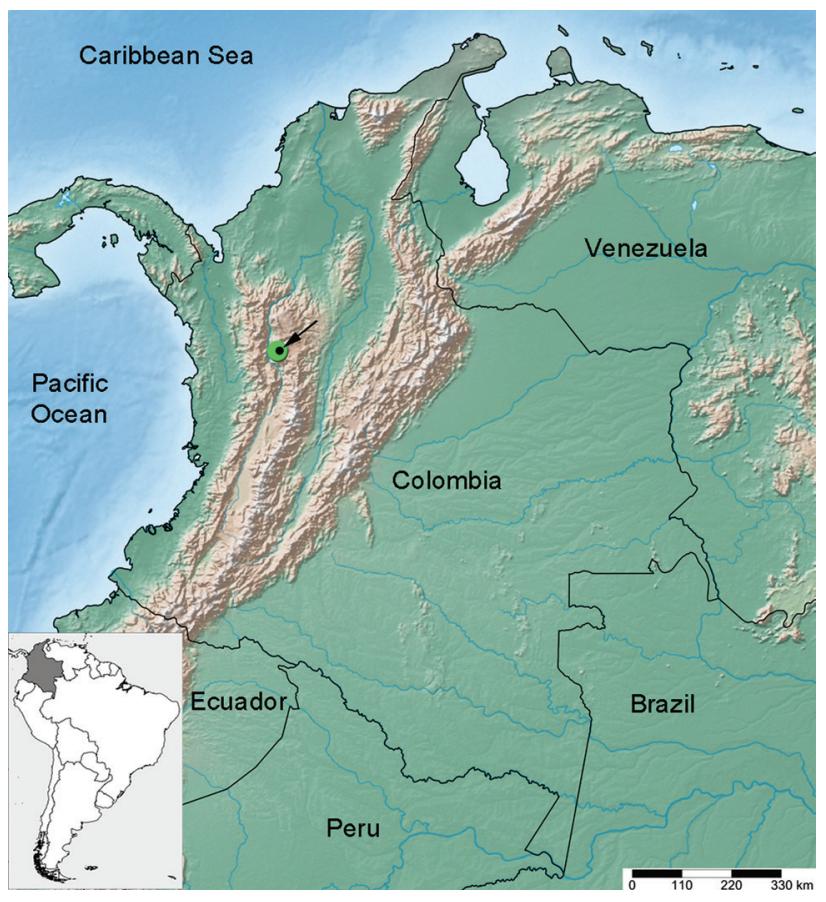

Figure 12. Geographic distribution of Pamphobeteus verdolaga sp. nov. Green circle shows distribution area of the species. Arrow points to Medellín city, Antioquia, Colombia.

broad embolus, $P$. antinous, $P$. crassifemur, $P$. grandis, $P$. verdolaga sp. nov., $P$. vespertinus and $P$. ultramarinus.

Additionally, the embolus presents apical, retrolateral and prolateral superior keels well developed. Pamphobeteus verdolaga sp. nov. is distinguished by having a poorly developed 
apical keel, and retrolateral keel length similar to apical keel. Pamphobeteus augusti, P. crassifemur, $P$. ferox and $P$. ornatus have prolateral inferior keel; it is poorly developed in the first two as in $P$. verdolaga sp. nov., and the last two have PI well developed. In the description of $P$. grandis (BERTANI et al. 2008: fig. 13) the apical keel was erroneously indicated as prolateral inferior keel.

Pamphobeteus females have similar spermathecae and are difficult to differentiate. They can be distinguished from other genera, except Xenesthis, by the presence of two elongated seminal receptacles, largely fused at the base. Pamphobeteus verdolaga sp. nov. is characterized by the very short oval seminal receptacles, which are curved toward the center.

Pamphobeteus petersi Schmidt, 2002 is not included in this discussion because this species is misplaced within Pamphobeteus. The copulatory organs of $P$. petersi resemble those of Megaphobema Pocock, 1901, thereby P. petersi must be transferred to Megaphobema. This transfer will be implemented and discussed within the context of a revision of the genus Pamphobeteus (in prep.).

\section{ACKNOWLEDGMENTS}

We thank the curators of the following institutions, who loaned specimens: Eduardo Flórez (ICN), Guillermo Castillo (UDENAR), Marta Wolff (CEUA), Alvaro Barragán (QCAZ), Rogerio Bertani (IBSP), Janet Beccaloni (BMNH), Christine Rollard (MNHN) and Peter Jäger (SMF). R. Gabriel and S. Esche provided additional illustrations and photographs of the type specimens; R. Bertani (IBSP) produced images of some species and critically reviewed the manuscript; Mathias Weibel helped with the English language of a previous version of this manuscript. Comments from three anonymous reviewers greatly improved the original ms. Conselho Nacional de Desenvolvimento Científico e Tecnológico (CNPq) provided support to YC through grant 190543/2015-9. Agencia Nacional de Investigación e Innovación (ANNI), Uruguay, supported CP through grant POS_NAC_2011_1_3624, and the German Academic Exchange Service (DAAD) provided a scholarship (Ref. 91625997). This research was partially funded with the Project CIQF-211 through the Comité para el Desarrollo de la Investigación (CODI-UdeA). The authors are also grateful to the Sostenibilidad program of the Universidad de Antioquia (UdeA), and Comité para el Desarrollo de la Investigación CONADI, Universidad Cooperativa de Colombia.

\section{LITERATURE CITED}

BERTANI R (2000) Male palpal bulbs and homologous features in Theraphosinae (Araneae, Theraphosidae). Journal of
Arachnology 28: 29-42.

Bertani R, Fukushima CS, da Silva Jr PI (2008) Two new species of Pamphobeteus Pocock 1901 (Araneae: Mygalomorphae: Theraphosidae) from Brazil, with a new type of stridulatory organ. Zootaxa 1826: 45-28.

Cooke JA, Roth VD, Miller F (1972) The Urticating Hairs of Theraphosidae. American Museum Novitates 2498: 1-43.

Estrada-Gómez S, Vargas Muñoz LJ, Quintana Castillo JC (2013) Extraction and partial characterization of venom from the Colombian spider Pamphobeteus aff. nigricolor (Aranae:Theraphosidae). Toxicon 76: 301-309.

Hamilton CA, Formanowicz DR, Bond JE (2011) Species Delimitation and Phylogeography of Aphonopelma hentzi (Araneae, Mygalomorphae,Theraphosidae): Cryptic Diversity in North AmericanTarantulas. Plos One 6: e26207. doi: 10.1371/ journal.pone.0026207

LONGHorn SJ, Nicholas M, ChUTER J, Vogler AP (2007) The utility of molecular markers from non-lethal dnasamples of the CITES II rotected "tarantula" Brachypelma vagans (araneae, theraphosidae). Journal of Arachnology 35: 278-292. doi: 10.1636/S05-62.1

Pérez-Miles F, Lucas SM, da Silva Jr PI, Bertani R (1996) Systematic revision and cladistic analysis of Theraphosinae (Araneae: Theraphosidae). Mygalomorph 1: 33-68.

Petrunkevitch A (1925) Arachnida from Panama. Transactions of the Connecticut Academy of Arts and Sciences 27: 51-248.

Shorthouse DP (2010) SimpleMappr: an online tool to produce publication-quality point maps. Available online at: http:// www.simplemappr.net [Accessed: 03/03/2016]

World Spider Catalog (2016) World Spider Catalog. Natural History Museum Bern, online at http://wsc.nmbe.ch, version 15.5 [Accessed: 03/03/2016]

Submitted: 21 June 2016

Received in revised form: 30 August 2016

Accepted: 3 September 2016

Editorial responsibility: Ricardo Pinto da Rocha

Author Contributions: YC, SEG, LJV and CP performed the field work and collected the specimens. YC and CP studied type material and other specimens, described and illustrated the new taxon. SEG and LJV carried out the molecular characterization. All authors discussed and wrote the paper.

Competing Interests: The authors have declared that no competing interests exist. 